\title{
Design issues, Topology issues, Quality of Service Support for Wireless Sensor Networks: Survey and Research Challenges
}

\author{
S.Muthukarpagam \\ IV B.E., Computer Science and \\ Engineering \\ J.J. College of engineering and \\ Technology, Tiruchirapalli, \\ Tamilnadu, INDIA
}

\author{
V.Niveditta \\ IV B.E, Computer Science and \\ Engineering, \\ J.J. college of Engineering and \\ Technology, Tiruchirapalli, \\ Tamilnadu, INDIA
}

\author{
S.Neduncheliyan \\ Department of Computer Science \\ and Engineering, \\ J.J. College of Engineering and \\ Technology, Tiruchirapalli, \\ Tamilnadu, INDIA
}

\begin{abstract}
Wireless Sensor Networks (WSN) are highly distributed self organized systems. WSN have been deployed in various fields. Because of some hardware problems, especially with respect to energy supply and miniaturization, WSN have certain short comings. This paper focuses on various issues such as routing challenges and design issues, topology issues and Quality of Service support issues associated with WSN. Design issues emphasis on designing the Wireless Sensor Networks in such a way that it should provide a fault tolerant communication with low latency. Topology issues include geographic routing, sensor hole problems and sensor coverage issues. Quality of Service aims at providing better networking services over current technologies
\end{abstract}

\section{Categories and Subject Descriptors}

C. Computer System Organization C.2 COMPUTERCOMMUNICATION NETWORKS, C.2.1 Network Architecture and Design. Wireless Communication

\section{General Terms}

Design and Reliability

\section{Keywords}

Latency, reliability, sensor holes, Quality of Service, Jitter, routing.

\section{INTRODUCTION}

In recent years, advances in miniaturization; low-power circuit design; simple, low power, yet reasonably efficient wireless communication equipment; and improved small-scale energy supplies have combined with reduced manufacturing costs to make a new technological vision possible: Wireless Sensor Networks (WSN). These Wireless Sensor Networks are highly distributed self-organized systems. WSNs provide a new paradigm for sensing and disseminating information from various environments, with the potential to serve many and diverse applications. With recent developments in the wireless networks field, new and innovative medical applications based on this technology are being developed in the research as well as commercial sectors. This paper discusses the various issues of WSN. The paper is organized as follows. Section II gives some related works with WSN. Section III describes the routing challenges and the design issues in WSN. Section IV discusses the various topology issues associated with WSN. Section V focuses on the quality of service Support (QoS) in WSN.

\section{RELATED WORKS}

A work titled "Rumor routing algorithm for sensor networks" explains the method for routing queries to nodes based on the event observed; not based on a unique id or geographic location of a node so that the data is allowed to be retrieved from the network keyed on the event and not on the underlying network addressing scheme or geography.[1]Another worker of WSN had worked for providing support for secure transient association between a master and a slave device or between peers in a Wireless ad-hoc network [2]. Many workers have worked on the security issues of WSN and one such work is "Talking to strangers: Authentication in adhoc wireless networks". It provides support for secure communication and authentication in wireless ad-hoc networks without any public key infrastructure. [3] Many researchers recognize the need for methods that deal with conflicting performance demands and set up a sensor network properly. Some authors suggest using a knowledge base to make a match between task-level demands and network protocols to use [8, 9]. A work on "Energy-efficient communication protocol for wireless micro sensor networks" presents a 2-level hierarchical routing protocol which attempts to minimize global energy dissipation and distribute energy consumption evenly across all nodes. [11]. A worker of WSN has worked on the need for robustness and Scalability, which leads to the design of localized algorithms, where sensors only interact with other sensors in a restricted vicinity and have at best an 
indirect global view.[13]. The research community generally ignores mobility in sensor-nets because sensor-nets were originally assumed to consist of static nodes. However, recent efforts such as RoboMote [15] and Parasitic-Mobility [16] have enabled mobility in sensor-nets.

\section{ROUTING CHALLENGES AND DESIGN ISSUES IINWSN}

\subsection{Fault -tolerant Communication}

Due to the deployment of sensor nodes in an uncontrolled or harsh environment, it is not uncommon for the sensor nodes to become faulty and unreliable [4]. This unreliability makes networks split then, some nodes cannot communicate other nodes. Thus, the framework can be resilient to the advent of faulty nodes.

\subsection{Low latency}

The events which the framework deals with are urgent which should be recognized immediately by the operator. Therefore, the framework has to detect and notify the events quickly as soon as possible.

\subsection{Management at a Distance}

Sensor nodes will be deployed at our-door field such as a subway station. It is difficult for managers or operators to manage the network directly. Thus the framework should provide an indirect remote control/ management system.

\subsection{Scalability}

A system, whose performance improves after adding hardware, proportionally to the capacity added, is said to be a scalable system. The number of sensor nodes deployed in the sensing area may be in the order of hundreds or thousands, or more. Any routing scheme must be able to work with this huge number of sensor nodes. Scalability in sensor networks can be measured in various dimensions

\subsection{Transmission Media}

In a multi-hop sensor network, communicating nodes are linked by a wireless medium. The traditional problems associated with a wireless channel (e.g., fading, high error rate) may also affect the operation of the sensor network.

\subsection{Coverage Problems}

One fundamental problem in wireless sensor networks is the coverage problem, which reflects the quality of service that can be provided by a particular sensor network. The coverage problem is defined from several points of view due to a variety of sensor networks and a wide-range of their applications.

\subsection{Network Scale and Time-Varying Characteristics of WSN}

Under severe energy constraints, Sensor nodes operate with limited computing, storage and communication capabilities [12]. Depending upon the application, the densities of the WSNs may vary widely, ranging from very sparse to very dense. In these sensor nodes the behavior of sensor nodes is dynamic and highly adaptive, as the need to self organize and conserve energy forces sensor nodes to adjust the behavior constantly in response to their current level of activity. Furthermore, the sensor nodes may be requires to adjust the behavior in response to the erratic and unpredictable behavior of wireless connections caused by high noise levels and radio-frequency interference, to prevent severe performance degradation of the application supported

\section{TOPOLOGY ISSUES OF WSN}

\subsection{Various issues}

Various topology issues such as geographic routing, sensor holes problems and Sensor Coverage Topology are discussed in this section.

\subsubsection{Geographic Routing}

Geographic routing is a routing principle that relies on geographic position information. It is mainly proposed for wireless networks and based on the idea that the source sends a message to the geographic location of the destination instead of using the network address.[6] Geographic routing uses geographic and topological information of the network to achieve optimal routing schemes with high routing efficiency and low power consumption

\subsubsection{Sensor Holes}

A routing hole consists of a region in the sensor network, where either nodes are not available or the available nodes cannot participate in the actual routing of the data due to various possible reasons.[7] If given a set of sensors and a target area, no coverage hole exists in the target area, if every point in the target area is covered by at least $\mathrm{k}$ sensors, where $\mathrm{k}$ is the required degree of coverage for a particular application. The identification of holes in a wireless sensor network is of primary interest since the breakdown of sensor nodes in a larger area often indicates one of the special events to be monitored by the network in the first place (e.g. outbreak of a fire, destruction by an earthquakes etc.). This task of identifying holes is especially challenging since typical wireless sensor networks consist of lightweight, low-capability nodes that are unaware of their geographic location

\subsubsection{Coverage Topology}

Coverage problem reflects how well an area is monitored or tracked by sensors. The coverage and connectivity problems in sensor networks have received considerable attention in the research community in recent years. This problem can be formulated as a decision problem, whose goal is to determine whether every point in the service area of the sensor network is covered by at least $k$ sensors, where $k$ is a given parameter

\subsection{Available Topologies}

We can use several network topologies to coordinate the WSN gateway, end nodes, and router nodes. Router nodes are similar to end nodes in that they can acquire measurement data, but we also can use them to pass along measurement data from other nodes. The first, and most basic, is the star topology, in which each node maintains a single, direct communication path with the 
gateway. This topology is simple but restricts the overall distance that the network can achieve. [5]

To increase the distance a network can cover, we can implement a cluster, or tree, topology. In this more complex architecture, each node still maintains a single communication path to the gateway but can use other nodes to route its data along that path. This topology suffers from a problem, however. If a router node goes down, all the nodes that depend on that router node also lose their communication paths to the gateway.

The mesh network topology remedies this issue by using redundant communication paths to increase system reliability. In a mesh network, nodes maintain multiple communication paths back to the gateway, so that if one router node goes down, the network automatically reroutes the data through a different path. The mesh topology, while very reliable, does suffer from an increase in network latency because data must make multiple hops before arriving at the gateway.

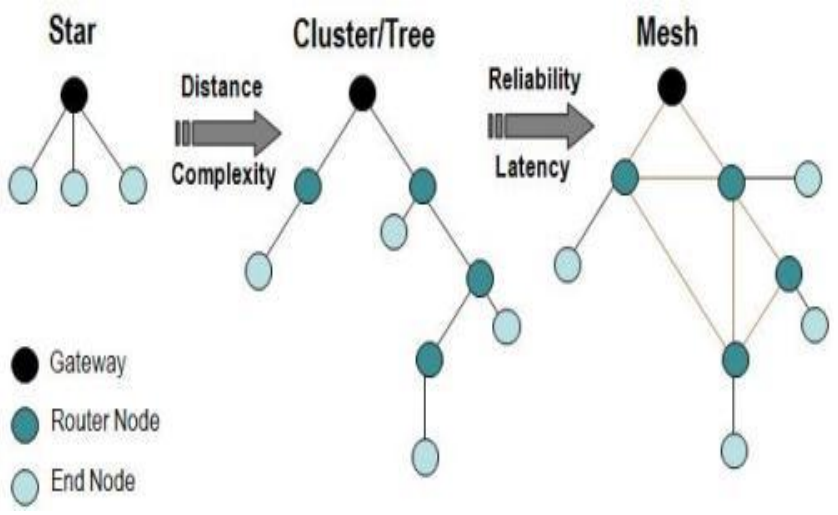

Figure 1. WSN Topologies

\section{QUALITY OF SERVICE (QOS) SUPPORT IN WSN}

Quality of Service (QoS) aims at providing better networking services over current technologies such as ATM, Ethernet and others. The main three parameters for QoS are latency (delay), jitter and loss. Other QoS parameters include reliability, responsiveness, mobility, power efficiency network availability and bandwidth

\subsection{Delay, Jitter and Loss}

Delay is the total amount of time a network spends to deliver a frame of data from source to destination. Jitter in turn is the delay between two consecutive packets in that frame. While loss determines the maximum amount of packets loss the stream can tolerate to provide good quality. Each parameter has been investigated thoroughly and many solutions are proposed such as forward error correction and interleaving [10].

\subsection{Reliability and Scalability}

In wireless sensor networks (or infrastructure less networks), reliability and scalability are always inversely coupled. In other words, it becomes more difficult to build a reliable ad hoc network as the number of nodes increases. This is due to the network overhead that comes with the increased size of the network. In ad hoc networks, the network is formed without any predetermined topology or shape. Therefore, any node wishing to communicate with other nodes should generate more packets than its data packets. These extra packets are generally called "control packets" or "network overhead." Route discovery packets and route response packets in typical ad hoc network routing protocols are a few examples of the overhead. As the size of the network grows, more control packets will be needed to find and keep the routing paths.

\subsection{Responsiveness}

Responsiveness is the ability of the network to quickly adapt itself to changes in topology. To achieve high responsiveness, an ad hoc network should issue and exchange more control packets, which will naturally result in less scalability and less reliability.

\subsection{Power Efficiency}

Power efficiency also plays another important role in this complex equation. A typical method for designing a low-power wireless sensor network is to reduce the duty cycle of each node. The drawback is that as the wireless sensor node stays longer in sleep mode to save power, there is less chance that the node can communicate with its neighbors. In addition to creating scalability challenges due to the need for a more complicated synchronization technique to keep more nodes in low duty cycle, this will decrease the network responsiveness and may also lower reliability due to the lack of the exchange of control packets and delays in packet delivery

\subsection{Mobility}

Mobility in sensor networks is highly essential for allowing communication between different connected components of the network. This also allows the operation of the sparse networks. When there is mobility in the sensor networks energy consumption is greatly reduced, so that the life time of the nodes are increased. Sensor mobility also allows better coverage

\subsection{Bandwidth}

Bandwidth is defined as the total distance or range between the highest and lowest signals on the communication channel. [14] Bandwidth represents the capacity of the connection. The greater the capacity, the more likely that greater performance will follow, though overall performance also depends on other factors, such as latency. Sensor networks need to be supplied with the required amount of bandwidth so that it is able to achieve a minimal required QoS. Limited bandwidth results in congestion which impacts normal data exchange and may also lead to packet loss.

\section{CONCLUSION}

Wireless sensor networks are more than just a specific form of ad hoc networks. Recent advanced hardware technologies result in more powerful sensors as small as a few millimeters volume. The main drawback is still energy constraints. Additional strategies aiming at extending sensor lifetimes have also been studied along with pre-processing or data aggregation prior to 
transmission, and the optimal positions to place sensors. The stringent miniaturization and cost requirements make economic usage of energy and computational power a significantly bigger issue than in normal ad hoc networks. As wireless sensor networks are still a young research field, much activity is still ongoing to solve many open issues. As some of the underlying hardware problems, especially with respect to the energy supply and miniaturization, are not yet completely solved, wireless sensor networks are having certain short comings, which are to be solved. WSN is emerging as a very important tool for making human life comfortable and safe. Yet, there is enormous scope for improving this WSN technology.

\section{REFERENCES}

[1]D. Braginsky, D. Estrin 2002. Rumor routing algorithm for sensor networks. International Workshop on Wireless Sensor Networks and Applications, WSNA

[2]F. Stajano and R. Anderson. 1999. The Resurrecting Duckling: Security Issues for Ad-hoc Wireless Networks wireless networks. 7th International Workshop on Security Protocols.

[3]D. Balfanz and D. Smetters and P. Stewart and H. Wong, 2002Talking to strangers: Authentication in adhoc wireless networks, Symposium on Network and Distributed Systems Security.

[4]I.F. Akyildiz, W. Su, Y. Sankarasubramaniam, and E. Cayirci, 2002 Wireless sensor networks: a survey, Computer Networks, vol 38, no. 4, pp. 393-422, (March 2002.)

[5]Mo Li, Baijian Yang, 2006 A Survey on Topology issues in Wireless Sensor Network International conference on wireless networks
[6]Pirmez, L., Delicato, F., Pires, P., Mostardinha, A., de Rezende, N.:2007 Applyingfuzzy logic for decision-making on wireless sensor networks. In: Fuzzy Systems Conference '07, Proc., IEEE (2007)

[7]Wolenetz, M., Kumar, R., Shin, J., Ramachandran, U.:2005 A simulation-based study of wireless sensor network middleware. Network Management 15(4) (2005)

[8]Kurose, J. F., and Ross, K. Computer Networking: A TopDown Approach Featuring the Internet. Addison-Wesley Longman Publishing Co., Inc., Boston, MA, USA,

[9]W.R. Heinzelman, A. Chandrakasan, H. Balakrishnan,"2000 Energy-ef_cient communication protocol for wireless microsensor networks", IEEE Hawaii International Conference on System Sciences,

[10]Kazem Sohraby, Daniel Minoli, Taieb F. Znati, "Wireless sensor networks: technology, protocols, and applications", A John Wiley \& Sons, Inc., Publication, 2007

[11]C. Intanagonwiwat and R. Govindan and D. Estrin "Directed Diffusion: A Scalable and Robust Communication" MOBICOM 2000

[12]K. Dantu, M. Rahimi, H. Shah, S. Babel, A. Dhariwal, and G. S. Sukhatme, "Robomote: Enabling mobility in sensor networks," in IEEE/ACM Fourth International Conference on Information Processing in Sensor Networks (IPSN/SPOTS), Apr. 2005

[13]M. Laibowitz and J. A. Paradiso, "Parasitic mobility for pervasive sensor networks," in Third International Conference on Pervasive Computing (PERVASIVE 2005), Munich, Germany, May 2005 